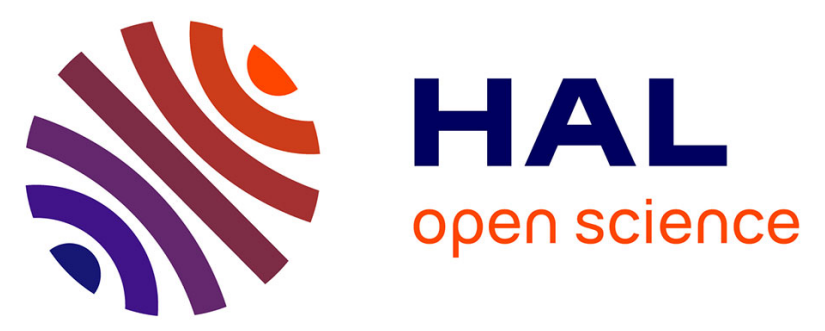

\title{
Multi-atlas-based segmentation of prostatic urethra from planning CT imaging to quantify dose distribution in prostate cancer radiotherapy
}

Oscar Acosta, Eugenia Mylona, Mathieu Le Dain, Camille Voisin, Thibaut Lizee, Bastien Rigaud, Carolina Lafond, Khemara Gnep, Renaud de Crevoisier

\section{To cite this version:}

Oscar Acosta, Eugenia Mylona, Mathieu Le Dain, Camille Voisin, Thibaut Lizee, et al.. Multiatlas-based segmentation of prostatic urethra from planning CT imaging to quantify dose distribution in prostate cancer radiotherapy. Radiotherapy \& Oncology, 2017, 125 (3), pp.492 - 499. 10.1016/j.radonc.2017.09.015 . hal-01671693

HAL Id: hal-01671693

https://hal-univ-rennes1.archives-ouvertes.fr/hal-01671693

Submitted on 16 Mar 2018

HAL is a multi-disciplinary open access archive for the deposit and dissemination of scientific research documents, whether they are published or not. The documents may come from teaching and research institutions in France or abroad, or from public or private research centers.
L'archive ouverte pluridisciplinaire HAL, est destinée au dépôt et à la diffusion de documents scientifiques de niveau recherche, publiés ou non, émanant des établissements d'enseignement et de recherche français ou étrangers, des laboratoires publics ou privés. 


\section{Multi-atlas-based segmentation of prostatic urethra from planning CT imaging to quantify dose distribution in prostate cancer radiotherapy}

Oscar Acosta ${ }^{1,2}$, Eugenia Mylona ${ }^{1,2}$, Mathieu Le Dain ${ }^{1,2}$, Camille Voisin ${ }^{1,2}$, Thibaut Lizee ${ }^{1,2}$, Bastien Rigaud $^{1,2}$, Carolina Lafond ${ }^{1,2,3}$, Khemara Gnep ${ }^{1,2,3}$, and Renaud de Crevoisier ${ }^{1,2,3}$

${ }^{1}$ INSERM U1099, Rennes, France

${ }^{2}$ Université de Rennes 1 - Laboratoire du Traitement du Signal et de l'Image, Rennes, France

${ }^{3}$ Centre Eugene Marquis, Département de Radiothérapie, Rennes, France

Running head: Multi-atlas urethra segmentation from planning CT

Keywords: prostate cancer radiotherapy, urinary toxicity, atlas-based segmentation, urethra segmentation, dose computation 


\begin{abstract}
Background and purpose: Segmentation of intra-prostatic urethra for dose assessment from planning CT may help explaining urinary toxicity in prostate cancer radiotherapy. This work sought to: i) propose an automatic method for urethra segmentation in CT, ii) compare it with previously proposed surrogate models and iii) quantify the dose received by the urethra in patients treated with IMRT.
\end{abstract}

Materials and methods: A weighted multi-atlas-based urethra segmentation method was devised from a training data set of 55 CT scans of patients receiving brachytherapy with visible urinary catheters. Leave-one-out cross validation was performed to quantify the error between the urethra segmentation and the catheter ground truth with two scores: the centerlines distance (CLD) and the percentage of centerline within a certain distance from the catheter (PWR). The segmentation method was then applied to a second test data set of 95 prostate cancer patients having received 78Gy IMRT to quantify dose to the urethra.

Results: Mean CLD was $3.25 \pm 1.2 \mathrm{~mm}$ for the whole urethra and $3.7 \pm 1.7 \mathrm{~mm}, 2.52 \pm 1.5 \mathrm{~mm}$, and $3.01 \pm 1.7 \mathrm{~mm}$ for the top, middle, and bottom thirds, respectively. In average, $53 \%$ of the segmented centerlines were within a radius $<3.5 \mathrm{~mm}$ from the centerline ground truth and $83 \%$ in a radius $<5 \mathrm{~mm}$. The proposed method outperformed existing surrogate models. In IMRT, urethra DVH was significantly higher than prostate DVH from V74Gy to V79Gy.

Conclusion: A multi-atlas-based segmentation method was proposed enabling assessment of the dose within the prostatic urethra. 


\section{Introduction}

In prostate cancer radiotherapy (PCRT), dose increase is limited by toxicity in organs at risk [1]. While gastrointestinal (GI) toxicity has been relatively reduced by the introduction of highly conformal image-guided radiotherapy techniques, genitourinary (GU) toxicity remains relatively stable, with a 5-year rate of Grade $\geq 2$ toxicity of approximately 15 to $20 \%$ [2, 3]. Urinary toxicity is a challenging issue, not only due to the variety of associated irritating or obstructive symptoms, but also owing to the limitations of dose descriptors and difficulties identifying the regions at risk responsible for those symptoms [4-6]. The bladder, for example, presents the largest inter-fraction shape variations, causing geometric and dose uncertainties that limit the possibility of accurately modeling the dose-volume response concerning GU toxicity [4, 7, 8, 9 ]. Most existing models address urinary toxicity by computing the dose to the bladder using either the dose parameters extracted from volumes (whole bladder or bladder wall), surface maps or localized sub-regions via spatial dose descriptors $[1,6,10,11,12,13,14]$ which partially have revealed global or local dose-effect relationships. Although there is evidence in prostate cancer brachytherapy that some urinary symptoms are related to urethra damage [15-17] this has not yet been shown in external-beam radiotherapy. Quantifying the delivered dose to the urethra may therefore improve our understanding of urinary toxicity or at least some of the related symptoms if we can accurately identify the organ from the planning CT. Thus, segmenting the urethra from the planning CT in order to assess the dose it receives, would pave the way for further studies on urinary toxicity prediction considering both the bladder and urethra.

To our knowledge, a formal segmentation of the intra prostatic urethra from CT images has not been addressed yet. However, indirect surrogate models for estimating the urethra position have been previously proposed $[18,19]$. They are nevertheless based on empirical considerations with respect to the prostate midplane. Segmenting the urethra from CT scans is fairly challenging. Not only is there already poor contrast between soft tissues like the prostate, bladder, and rectum, thus rendering segmentation difficult for planning, but the intra-prostatic urethra itself is completely invisible. These issues restrict the use of classic intensity-based 
segmentation methods. Atlas-based approaches, widely discussed in the literature [20-26], are common methods for organ segmentation. In atlas-based methods, precomputed segmentation in a template space is propagated onto the image to be segmented via spatial normalization (registration) as depicted in Figure A1 (supplemental material). Several individuals from a population can be used to constitute the atlas (multi-atlas). This allows to overcome the interindividual variability and registration issues. Previous works have shown the benefits of combining multiple atlases in improving segmentation accuracy [20, 22, 23, 27-29].

In this paper, we propose a weighted Multi-Atlas-Based Urethra Segmentation strategy, herein called MABUS, from planning CTs. Our goal is to provide a method that could be applied to a different set of patients receiving external-beam radiotherapy in order to assess the dose to the urethra and relate with toxicity effects.

\section{Material and methods}

This study is divided into three main parts: i) a description of MABUS, the multi-atlas-based urethra segmentation method which illustrates the whole implemented framework, from the atlas construction to the final urethra segmentation, ii) the evaluation of the method's accuracy with respect to the urethra ground truth in a leave one out cross validation framework, and comparison with the existing surrogates proposed by Bucci [18] and Waterman [19] and finally iii) the computation of the dose received by the urethra in a different series of patients with prostate cancer IMRT which aims to introduce the way in which the method may be used in toxicity studies. 


\section{Multi-atlas based urethra segmentation (MABUS) method description}

In general, as depicted in Figure A1 in supplemental material atlas-based segmentation relies on the registration of a template ${ }^{I_{i}}$ to the query image $I_{q}$, in order to obtain a transformation $T_{I_{i} \rightarrow I_{q}}$, which maps a set of generated labels $\zeta_{i}$ onto $I_{q}$. If the mapping is anatomically correct, the yielded segmentation is accurate and anatomically meaningful. Multi-Atlas based segmentation builds upon this idea by extending the number of atlases thereby reducing the interindividual variability issues.

Following the multi-atlas concept, the proposed MABUS was devised and can be divided into seven steps as depicted in Figure 1. In summary, an atlas dataset was first built from manuallydelineated CTs including the urethra, thanks to the presence of an urinary probe (Step 1). The query image to be segmented $I_{q}$ was then rigidly aligned with the same template $I_{T}$ as the atlas

database (Step 2) and features were extracted $F_{q}=\left\{f_{1 q}, . ., f_{5 q}\right\}$ (Step 3). By comparing the features, the atlases were ranked according to their similarity to the query image (Step 4). The labels $\zeta_{i}=\{$ urethra $\}$ from the top $n=10$ ranked atlases were then propagated to the query image using an accurate non-rigid registration method (Step 5) designed to match the prostate anatomies. Finally, the urethra segmentation was obtained by combining different labels in a weighted-fusion process (Step 6), followed by centerline detection (Step 7). The image segmentation methods were developed in C++ using the Insight Toolkit libraries (ITK) [30] and python [31] open source technologies. We made the atlas database as well as the whole code available for further studies.

[Figure 1 here] 


\section{Atlas building from training data (Step 1)}

For the atlas building, we used an initial series of CT scans $(512 \times 5120.63 \times 0.63 \mathrm{~mm}$ axial pixels and $3 \mathrm{~mm}$ slices) from 55 patients treated for localized prostate cancer with Iodine-125 brachytherapy. All the patients were fitted with urinary catheters, enabling urethra segmentation. The prostate, bladder, and urethra were delineated for each by the same radiation oncologist, constituting the set of atlases $I_{i}$, with the label $\zeta_{i}=\{u r e t h r a\}$.

\section{Template selection and rigid registration (Step 2)}

A first average patient $I_{T}$ was selected as a common coordinate system. This patient was the closest to all others in terms of prostate volume. The whole population was then rigidly registered to this patient by aligning the prostate centroids followed by a fine alignment of bony anatomy. This enabled geometrical descriptors to be generated and compared in the same common space. The central lines of the manually delineated catheters were computed by extracting their centroid at each slice.

\section{Feature extraction (Step 3)}

A simplified geometrical description of the anatomy (prostate/bladder) was generated to characterize each individual. Hence, the obtained vector $F_{i \in[1, . ., N]}=\left\{f_{1 i}, . ., f_{5 i}\right\}$ describes the individuals in terms of i) prostate volume ii) distance between prostate and bladder centroids, iii) the extension of the bladder in the anterior posterior direction, and iv) the orientation of the bladder with respect to the prostate centroid, regarding two angles $(\varphi)$ and $(\theta)$, which 
respectively describe the anteroposterior and lateral directions. The descriptors were then normalized across the population with a z-score.

\section{Query image and atlas selection (Step 4)}

For an image $I_{q}$ to be segmented, the two previously described steps (2 and 3) were applied as for the training database. Thus, rigid registration to the common template $I_{T}$ and characterization yield for $I_{q}$ the vector $F_{q}=\left\{f_{1 q}, . ., f_{5 q}\right\}$, exhibiting similar features as computed for the atlas dataset. Following z-score normalization, the Euclidean distances $d_{i-q}=\left\|F_{i}-F_{q}\right\|$ between features enabled the individuals from the atlas $I_{i}$ to be ranked in terms of similarity to the query image $I_{q}$. In our multi-atlas strategy, only the top $(n=10)$ ranked atlases were selected as the closest to the query image $I_{q}$, with all remaining atlases discarded. Since their configuration is similar to that of the query image, the urethra is expected to lie inside the prostate in a similar position. The number of atlases $(n=10)$ was selected as a tradeoff between computational time and optimized results in a leave-one-out segmentation process in which the top $1,2, . ., n$ atlases were tested.

\section{Non-rigid registration (Step 5)}

In this step, the labels $\zeta_{i}$ from the $n$ most suitable previously-selected atlases were non-rigidly propagated to the prostate of the query image. To register each prostate from the best atlases to the prostate of the query image $I_{q}$ we applied a Laplacian-based registration method, built upon our previous work [32], but here only considering the prostate. In our implementation, instead of using the central line, we selected the centroid $C p$ of the prostate for computing a scalar field 
$u(x, y, z)$ by applying Laplace's equation inside the prostate volume, demarcated by an external boundary $F_{E x t}$, here the prostate surface, and an internal boundary, here the prostate centroid Cp, as:

$$
\Delta u(x, y, z)=\frac{\partial^{2} u}{\partial x^{2}}+\frac{\partial^{2} u}{\partial y^{2}}+\frac{\partial^{2} u}{\partial z^{2}}=0
$$

with $u(x, y, z)=u_{E x t}$, if $(x, y, z) \in F_{E x t}$ and $u(x, y, z)=u_{C p}$, if $(x, y, z) \in C p$, where $\left\{u_{E x t}, u_{C p}\right\} \in$ $\mathbb{R}$. This scalar field $u$ provides a structural and normalized descriptor to be exploited in a Demons-based non-rigid registration framework [33]. This step yields a 3D deformation field (DF) as a set of 3D vectors describing the voxel-wise deformation of each of the atlas images to $I_{q}$. The binary labels $\zeta_{i}=\{$ urethra $\}$ from the selected atlases were then propagated to the query image space using the calculated transformation $T=D F \circ T_{\text {Rigid }}$ and nearest-neighbor interpolation in order to preserve the binary nature of the propagated labels.

\section{Weighted-label fusion (Step 6)}

Once the labels were propagated to the same coordinate system, namely the prostate of the query image $I_{q}$, the raised question was how to fuse all the warped labels $\zeta_{i}=\{$ urethra $\}$ to yield the best segmentation result. Different decision rules may be applied, such as a simple voting-rule [34], a weighted decision based on similarity [35] or a Bayesian approach, such as the simultaneous truth and performance level estimation (STAPLE) [28]. We opted for the weighted-label fusion approach, resulting in the following probability map:

$$
p_{\zeta_{i}}=\frac{1}{N} \sum_{i=1}^{N} w_{i} \zeta_{i}
$$

with the weights $w$ derived as a function of the Euclidean distance between geometric 
features, $d_{i-q}=\left\|F_{i}-F_{q}\right\|$, giving

$$
w_{i}=\frac{e^{-d_{i-q}}}{\sum_{i=1}^{N} d_{i-q}}
$$

Thus, the contribution of each non-rigidly propagated label to this map heavily depended on the similarity between the atlas and the query image.

\section{Centerline and urethra detection (Step 7)}

A $50 \%$ threshold was applied to the probability map $p_{\zeta_{i}}$, followed by a centerline computation as a 3D cubic spline curve of equidistant points. This resulted in a smooth path $\gamma_{q}$ describing the urethra within the prostate. Considering the urethra to be a tubular-like structure, the final urethral region $\zeta_{q}$ was obtained as a $5 \mathrm{~mm}$ zone around $\gamma_{q}$.

\section{Evaluation of the accuracy of the segmentation method}

Leave-one-out cross validation was performed to evaluate the accuracy of the proposed method. Thus, each of the individuals from the atlas database was iteratively selected as query image $I_{q}$ and the resulting segmentations compared with the catheter positions. For our purposes, the central path described by the catheter was considered the ground truth. 
Two metrics were used to evaluate the method's accuracy. A score based on the centerline distance (CLD) was devised to assess the differences between the obtained discretized urethra path $\gamma_{q}$ and the catheter central line $\rho_{G T}$ as follows:

$$
\operatorname{CLD}\left(\gamma_{q}, \rho_{G T}\right)=\frac{1}{K} \sum_{k=1}^{K} \operatorname{dist}\left(\gamma_{q}^{k}, \rho_{G T}\right)
$$

where $\gamma_{q}^{k}$ represents the $k^{\text {th }}$ point of the urethra central line, dist $\left(\gamma_{q}^{k}, \rho_{G T}\right)$ is the Euclidean distance of this point to the catheter central line, and $K$ is the number of points. Figure 3 illustrates the way in which the CLD was computed from this distance map. This score was computed for the whole segmented path $\gamma_{c}$, as well as for each region produced after splitting the central path ground truth into three equivalent segments (from the apex to base). The percentage of the points from $\gamma_{c}$ lying inside a region around the centerline ground truth (PWR) within $\quad 3.5 \mathrm{~mm}$ and $5 \mathrm{~mm}$ radius were also quantified. For comparison with the existing surrogates, we implemented the methods proposed by Bucci [18] (deviated surrogate) and Waterman [19] (centered surrogate) which are based on the geometrical center of the axial midplane.

\section{Dosimetric study: assessment of the dose received by the urethra in prostate cancer IMRT}

\section{Data}

A second series of 95 patients having received 78Gy IMRT for prostate cancer between July 2012 and June 2015 were analyzed. The target volume included the prostate, sparing the pelvic lymph nodes. Target volumes and organs at risks (bladder, prostate) were delineated on CT slices according to the French GETUG group recommendations [36]. IMRT combined with image-guided radiation therapy (IGRT) were used to deliver a total dose of 78Gy for cone-beam CT or 80Gy for fiducials to the prostate over eight weeks at $2 \mathrm{~Gy} /$ fraction. The pelvic lymph 
nodes were not irradiated. Bladder dose-volume histograms (DVHs) complied with GETUG recommendations, namely V70 $<50 \%$.

\section{Urethra segmentation and dose comparison}

The proposed segmentation method was applied to patients treated with IMRT. Manual delineations of the prostate and bladder were used to automatically segment the urethra within a $5 \mathrm{~mm}$ diameter along the centerline. The dose within the urethra was assessed from the 3D planning dose distribution and compared to the dose to the prostate. A comparison between the prostate and urethra DVHs was performed using a Wilcoxon non parametric test.

\section{Results}

\section{Atlas construction}

The inter-individual variability found in terms of feature descriptors $F_{i \in[1, \ldots, N]}=\left\{f_{1 i}, \ldots, f_{5 i}\right\}$ was very high. The features represented in average $62.58 \pm 15 \mathrm{cc}$ for the prostate volume, $43.13 \pm 15$ $\mathrm{mm}$ for the distance between prostate and bladder centroids, $19.3 \pm 19 \mathrm{~mm}$ for the bladder extension in the horizontal/y- axis and $58.86 \pm 19.46(\varphi)$ and $120.47 \pm 61.5(\theta)$ for the angles describing respectively the antero-posterior and lateral directions of the bladder with respect to the prostate centroid. Figure 2 displays the distribution of the interindividual normalized distance across individuals in the z-score space.

[Figure 2 here]

\section{Urethra segmentation accuracy}


Leave one out results and comparisons with Waterman's and Bucci's are shown in Figure 3. In average MABUS outperformed the other two surrogate models. Global CLD was $3.25 \pm 1.2$ with MABUS, while $6.11 \pm 1.96$ with Waterman and $3.91 \pm 1.46$ with Bucci's method $(p<0.001)$. Considering the urehtra by thirds, with MABUS the computed CLD scores were $3.67 \pm 1.66 \mathrm{~mm}$, $2.52 \pm 1.58 \mathrm{~mm}$ and $3.01 \pm 1.76 \mathrm{~mm}$ for the top middle and bottom thirds respectively. Significantly better than the other models: $10.23 \pm 4.05 \mathrm{~mm}, 3.86 \pm 1.89 \mathrm{~mm}$ and $3.64 \pm 2.23$ for Waterman and $5.92 \pm 2.81,3.39 \pm 1.69$ and $3.64 \pm 2.23$ for Bucci. Likewise for the portion of the central line (PWR) within the $3.5 \mathrm{~mm}$ and $5 \mathrm{~mm}$ radius regions: $0.53 \pm 0.29$ and $0.83 \pm 0.18$ respectively for MABUS with a maximum of 1 in both cases. With Waterman we obtained $0.32 \pm 0.19$ and $0.53 \pm 0.17(\mathrm{p}<0.001)$ and with Bucci $0.51 \pm 0.22(\mathrm{p}=0.5)$ and $0.74 \pm 0.21(\mathrm{p}<0.01)$. Figure 4 depicts those results. There were some outliers for whom the segmentation was not as good as expected. Those outliers appear as dissimilar to the remaining population as shown in Figure 2. Only 3 segmentations out of 54 presented a score inferior to 0.5 in the $5 \mathrm{~mm}$ region. Figure 5 shows six examples of resulting segmentations overlaid on the manually delineated catheters. Top row illustrates a comparison of the proposed method with the surrogate models Figure 5 a) Waterman, b) Bucci and c) MABUS. Low row shows different results with MABUS on different individuals. It can be observed the complimentary information brought by the two different scores. For instance, in case of Figure $5 \mathrm{~d}$ ) the CLD was $3.4 \mathrm{~mm}$ and the whole centerline was within the first $5 \mathrm{~mm}$ region (PWR). For c) CLD $=2.8 \mathrm{~mm}$. It is worth noting that although only half of the points where within the first $3.5 \mathrm{~mm}$, the whole centerline was within the first $5 \mathrm{~mm}$.

[Figure 3 here]

[Figure 4 here]

[Figure 5 here] 


\section{Dosimetric study}

Urethra and prostate DVHs were significantly different. The bin-wise DVH comparison show that the volume (\%) receiving a dose between D74Gy to D79Gy by the urethra was significantly higher than in the prostate $(\mathrm{p}<0,01)$. Figure A3 in supplemental material highlights the prostate and urethra DVH bins, where statistically significant differences are represented by the red circles. Figure 6 shows an example of 3D dose distribution within the manually segmented volumes for planning together with the automatically segmented urethra. Considering the urethra, the prostate and the PTV , it can be seen that high doses appear in the urethra (iso-dose curves and DVH values $>$ V70). During the 3D dose optimization, the constraints to the PTV and to the organs at risk, bladder and rectum, will introduce a high dose gradient close to the PTV. Although the PTV is well covered it will receive $90 \%$ of the dose, whereas the urethra being central to the prostate will likely receives the higher doses.

[Figure 6 here]

\section{Discussion}

We proposed a weighted multi-atlas based method to segment the intra-prostatic urethra from planning CT and compared with two previously surrogate models based on the central axis of the prostate, Waterman et al. [19] (centered surrogate) and Bucci et al. [18] (deviated surrogate). The method does not need any catheter to estimate the position of the urethra as it is based on the combination of similar cases contained in a large data set of atlases, which are 
weighted and combined to achieve an accurate segmentation The obtained accuracy of the urethra segmentation considering the CLD (in average $3.25 \mathrm{~mm}$ ), computed in a leave one out cross-validation enabled to assess dose to the urethra in a different IMRT database . With our method, the measured dose received by the urethra appears slightly higher than the dose received by the whole prostate. This is likely due to the position of the urethra rather central within the prostate, relatively far from the rectum where a gradient of dose appears (Figure 6). Such findings support the use of our urethra segmentation method to potentially improve urinary toxicity prediction by considering both the dose received by the urethra and the prostate.

To our knowledge there is no evidence in the literature of any method for explicitly segmenting the urethra in the planning CTs. An atlas based urethra segmentation method in MRI was proposed [37], within a SBRT perspective, but without a formal segmentation propagation towards the planning CT. The first method proposed by Waterman to estimate the dose to the urethra from CT scan appears in brachytherapy [19]. As mentioned before, in that study, the urethra was estimated as a geometric surrogate based upon the prostate centerline. They found a good correspondence of the urethral doses (D10, D25 and D50) between this model and the urinary catheter. This model was latterly evaluated in brachytherapy patients with visible catheter [38]. They showed that a surrogate defined at the geometric center of the prostate may significantly overestimate the dose to the urethra. A surrogate urethra model by considering a slight deviation of 30 degrees anteriorly with respect to the central axis was proposed by Bucci et al. [18]. This deviation provided a better dose estimate than Waterman [19]. Here, we evaluated these two urethral surrogates with the same dataset. Compared to them our method performed better in the same regions (overall CLD $=3.25 \pm 1.2 \mathrm{~mm}$ with MABUS vs $3.91 \pm 1.46$ with the deviated surrogate and $6.11 \pm 1.96 \mathrm{~mm}$ with the centered surrogate). The difference with the central axis is less pronounced in the bottom third, suggesting that a good approximation of the urethra in this region may be achieved. However in the upper part, although the slight deviation of 30 degrees offers a good approximation for some individuals, this is not the case for all the 
patients. Our multi-atlas approach enabled to devise a strategy aimed at finding the $n$ most suitable atlas within the dataset by defining a similarity metric based on simple prostate and bladder geometric features. The prostate and bladder segmentations were used as they are generated during the dose planning clinical protocol. Considering the proposed features, the large interindividual variability was captured as shown in the similarity map (Figure 2). Thus, with the exception of some outliers, one can find good candidates in the atlas selection procedure for each considered individual. The atlas selection step is indeed crucial to accurately segment the urethra as demonstrated in the leave one out experiments. With the proposed features, it has been shown that a trend arises when correlating similarity metric (distance) and segmentation outcome. As shown in figure A2(Supplemental material), more similar atlas yields a better segmentations if only one single atlas was used for segmentation . Then, adding multiple atlases improves the accuracy when fusing labels from the closest atlas [29]. Other global or local features based on CT intensity or shape descriptors may have been proposed (mutual information, cross correlation, SPHARM, etc.), but with a limitation concerning accuracy assessment due to the presence of the catheter. The fusion step takes into account the interindividual similarity by weighting the contribution to the probability map via an exponential function, which has been shown to be more performant than simple averaging [35]. This strategy led us to limit to 10 , the number of selected atlas, as by adding more their contribution is vanished in an exponential function. Highly contributive is the prostate non-rigid registration based on the Laplacian scalar field [32]. Indeed, the main feature brought by the Laplacian is the computation of a normalized structural description comparable across individuals, as opposed to classical distance maps.

We used for evaluation the scores based on distance centerlines as proposed by [38] as the urethra is considered as a path within a tiny tube-like structure. Other scores based on volume overlap (Dice, Jaccard) are not suited here for assessing segmentation accuracy. The obtained scores enable the method to be used within a perspective of assessing dose to the urethra 
within an acceptable margin. However, improvements may be done within a perspective of IMRT planning and dose escalation. The atlas segmentation method may exhibit some limitations if we consider the hypothesis of non-preservation of the urethra shape with and without a catheter. One way to demonstrate that hypothesis will be the MRI where the urethra might be visible. However, in brachytherapy studies the urethra position is only given by the catheter [15-17]. Further limitations concerns the patient variability in IMRT patients compared to the atlas database as prostate and bladder volumes may be higher because of the patient selection and the presence of a catheter.

When applying the proposed method to an independent data set of patients treated with IMRT, a large difference was found in the dose to the urethra (higher doses) compared to the prostate. These findings suggest further studies to be performed on urinary toxicity by quantifying the dose to the urethra as reported in brachytherapy [15-17]. New multimodal models combining also dose to the bladder with clinical factors, biological parameters and other multimodal data within a radiomics framework could provide new insights into the urinary toxicity

\section{Acknowledgments}

This work received a French Government Grant (through the CominLabs excellence laboratory and managed by the National Research Agency in the "Investing for the Future" program, under reference ANR-10-LABX-07-01). 


\section{References}

[1]. Fiorino, C., R. Valdagni, T. Rancati, and G. Sanguineti, Dose-volume effects for normal tissues in external radiotherapy: pelvis. Radiother Oncol, 2009. 93(2): p. 153-67.

[2]. Michalski, J.M., Y. Yan, D. Watkins-Bruner, et al., Preliminary toxicity analysis of 3-dimensional conformal radiation therapy versus intensity modulated radiation therapy on the high-dose arm of the Radiation Therapy Oncology Group 0126 prostate cancer trial. Int J Radiat Oncol Biol Phys, 2013. 87(5): p. 932-8.

[3]. Byrne, K., G. Hruby, A. Kneebone, et al., Late Genitourinary Toxicity Outcomes in 300 Prostate Cancer Patients Treated With Dose-escalated Image-guided Intensity-modulated Radiotherapy. Clin Oncol (R Coll Radiol), 2017.

[4]. Yahya, N., M.A. Ebert, M. Bulsara, et al., Independent external validation of predictive models for urinary dysfunction following external beam radiotherapy of the prostate: Issues in model development and reporting. Radiother Oncol, 2016. 120(2): p. 339-45.

[5]. Yahya, N., M.A. Ebert, M. Bulsara, et al., Statistical-learning strategies generate only modestly performing predictive models for urinary symptoms following external beam radiotherapy of the prostate: A comparison of conventional and machine-learning methods. Med Phys, 2016. 43(5): p. 2040.

[6]. Thor, M., C. Olsson, J.H. Oh, et al., Urinary bladder dose-response relationships for patient-reported genitourinary morbidity domains following prostate cancer radiotherapy. Radiother Oncol, 2016. 119(1): p. 117-22.

[7]. Thor, M., A. Apte, J.O. Deasy, et al., Dose/volume-response relations for rectal morbidity using planned and simulated motion-inclusive dose distributions. Radiother Oncol, 2013. 109(3): p. 388-93.

[8]. Thor, M., A. Apte, J.O. Deasy, and L.P. Muren, Statistical simulations to estimate motion-inclusive dose-volume histograms for prediction of rectal morbidity following radiotherapy. Acta Oncol, 2013. 52(3): p. 666-75.

[9]. O'Callaghan, M.E., E. Raymond, J.M. Campbell, et al., Patient-Reported Outcomes After Radiation Therapy in Men With Prostate Cancer: A Systematic Review of Prognostic Tool Accuracy and Validity. Int J Radiat Oncol Biol Phys, 2017. 98(2): p. 318-337.

[10]. Palorini, F., C. Cozzarini, S. Gianolini, et al., First application of a pixel-wise analysis on bladder dosesurface maps in prostate cancer radiotherapy. Radiother Oncol, 2016. 119(1): p. 123-8.

[11]. Improta, I., F. Palorini, C. Cozzarini, et al., Bladder spatial-dose descriptors correlate with acute urinary toxicity after radiation therapy for prostate cancer. Phys Med, 2016. 32(12): p. 1681-1689.

[12]. Bagala, P., G. Ingrosso, M.D. Falco, et al., Predicting genitourinary toxicity in three-dimensional conformal radiotherapy for localized prostate cancer: A dose-volume parameters analysis of the bladder. J Cancer Res Ther, 2016. 12(2): p. 1018-24.

[13]. Yahya, N., M.A. Ebert, M.J. House, et al., Modeling Urinary Dysfunction After External Beam Radiation Therapy of the Prostate Using Bladder Dose-Surface Maps: Evidence of Spatially Variable Response of the Bladder Surface. Int J Radiat Oncol Biol Phys, 2017. 97(2): p. 420-426.

[14]. Yahya, N., M.A. Ebert, M. Bulsara, et al., Urinary symptoms following external beam radiotherapy of the prostate: Dose-symptom correlates with multiple-event and event-count models. Radiother Oncol, 2015. 117(2): p. 277-82.

[15]. Earley, J.J., A.M. Abdelbaky, M.J. Cunningham, et al., Correlation between prostate brachytherapyrelated urethral stricture and peri-apical urethral dosimetry: a matched case-control study. Radiother Oncol, 2012. 104(2): p. 187-91.

[16]. Thomas, C., M. Keyes, M. Liu, and V. Moravan, Segmental urethral dosimetry and urinary toxicity in patients with no urinary symptoms before permanent prostate brachytherapy. Int J Radiat Oncol Biol Phys, 2008. 72(2): p. 447-55.

[17]. Merrick, G.S., W.M. Butler, K.E. Wallner, et al., Risk factors for the development of prostate brachytherapy related urethral strictures. J Urol, 2006. 175(4): p. 1376-80; discussion 1381. 
[18]. Bucci, J., I. Spadinger, M. Hilts, et al., Urethral and periurethral dosimetry in prostate brachytherapy: is there a convenient surrogate? Int J Radiat Oncol Biol Phys, 2002. 54(4): p. 1235-42.

[19]. Waterman, F.M. and A.P. Dicker, Determination of the urethral dose in prostate brachytherapy when the urethra cannot be visualized in the postimplant CT scan. Med Phys, 2000. 27(3): p. 448-51.

[20]. Commowick, O., V. Gregoire, and G. Malandain, Atlas-based delineation of lymph node levels in head and neck computed tomography images. Radiother Oncol, 2008. 87(2): p. 281-9.

[21]. Martin, S., V. Daanen, and J. Troccaz, Atlas-based prostate segmentation using an hybrid registration. International Journal of Computer Assisted Radiology and Surgery, 2008. 3(6): p. 485492.

[22]. Aljabar, P., R.A.A. Heckemann, A. Hammers, J.V.V. Hajnal, and D. Rueckert, Multi-atlas based segmentation of brain images: Atlas selection and its effect on accuracy. Neurolmage, 2009. 46(3): p. 726-738.

[23]. Langerak, T.R., U.A. van der Heide, A.N.T.J. Kotte, et al., Label Fusion in Atlas-Based Segmentation Using a Selective and Iterative Method for Performance Level Estimation (SIMPLE). IEEE Transactions on Medical Imaging, 2010. 29(12): p. 2000-2008.

[24]. Martin, S., J. Troccaz, and V. Daanenc, Automated segmentation of the prostate in 3D MR images using a probabilistic atlas and a spatially constrained deformable model. Medical Physics, 2010. 37(4): p. 1579-1590.

[25]. Ramus, L., J. Thariat, P.Y. Marcy, et al., Automatic segmentation using atlases in head and neck cancers: Methodology. Cancer Radiothérapie 2010. 14(3): p. 206-12.

[26]. van Rikxoort, E.M., M. Prokop, B. de Hoop, et al., Automatic segmentation of pulmonary lobes robust against incomplete fissures. IEEE Transactions on Medical Imaging, 2010. 29(6): p. 1286-96.

[27]. Rohlfing, T., R. Brandt, R. Menzel, and C.R. Maurer, Evaluation of atlas selection strategies for atlasbased image segmentation with application to confocol microscopy images of bee brains. Neuroimage, 2004. 21(4): p. 1428-1442.

[28]. Warfield, S.K., K.H. Zou, and W.M. Wells, Simultaneous truth and performance level estimation (STAPLE): an algorithm for the validation of image segmentation. IEEE Transactions on Medical Imaging, 2004. 23(7): p. 903-921.

[29]. Acosta, O., J. Dowling, G. Drean, et al., Multi-Atlas-Based Segmentation of Pelvic Structures from CT Scans for Planning in Prostate Cancer Radiotherapy, in Abdomen and Thoracic Imaging: An Engineering \& Clinical Perspective, A.S. El-Baz, L. Saba, and J. Suri, Editors. 2014, Springer US: Boston, MA. p. 623-656.

[30]. McCormick, M., X. Liu, J. Jomier, C. Marion, and L. Ibanez, ITK: enabling reproducible research and open science. Front Neuroinform, 2014. 8: p. 13.

[31]. PSF. Python, open source techology. 2017; Available from: https://www.python.org/psf-landing/.

[32]. Dréan, G., O. Acosta, C. Lafond, et al., Interindividual registration and dose mapping for voxelwise population analysis of rectal toxicity in prostate cancer radiotherapy. Medical Physics, 2016. 43(6): p. 2721-2730.

[33]. Wang, H., L. Dong, M.F. Lii, et al., Implementation and validation of a three-dimensional deformable registration algorithm for targeted prostate cancer radiotherapy. Int J Radiat Oncol Biol Phys, 2005. 61(3): p. 725-35.

[34]. Acosta, O., A. Simon, F. Monge, et al. Evaluation of multi-atlas-based segmentation of CT scans in prostate cancer radiotherapy. in Biomedical Imaging: From Nano to Macro, 2011 IEEE International Symposium on. 2011.

[35]. Isgum, I., M. Staring, A. Rutten, et al., Multi-atlas-based segmentation with local decision fusionApplication to cardiac and aortic segmentation in CT scans. IEEE Transactions on Medical Imaging, 2009. 28(7): p. 1000-10.

[36]. Beckendorf, V., S. Guerif, E. Le Prise, et al., 70 Gy versus 80 Gy in localized prostate cancer: 5-year results of GETUG 06 randomized trial. Int J Radiat Oncol Biol Phys, 2011. 80(4): p. 1056-63.

[37]. Kataria, T., D. Gupta, S. Goyal, et al., Simple diagrammatic method to delineate male urethra in prostate cancer radiotherapy: an MRI based approach. Br J Radiol, 2016. 89(1068): p. 20160348.

[38]. Lee, H.K., W.D. D'Souza, J.M. Yamal, et al., Dosimetric consequences of using a surrogate urethra to estimate urethral dose after brachytherapy for prostate cancer. Int J Radiat Oncol Biol Phys, 2003. 57(2): p. 355-61. 


\section{Figures}

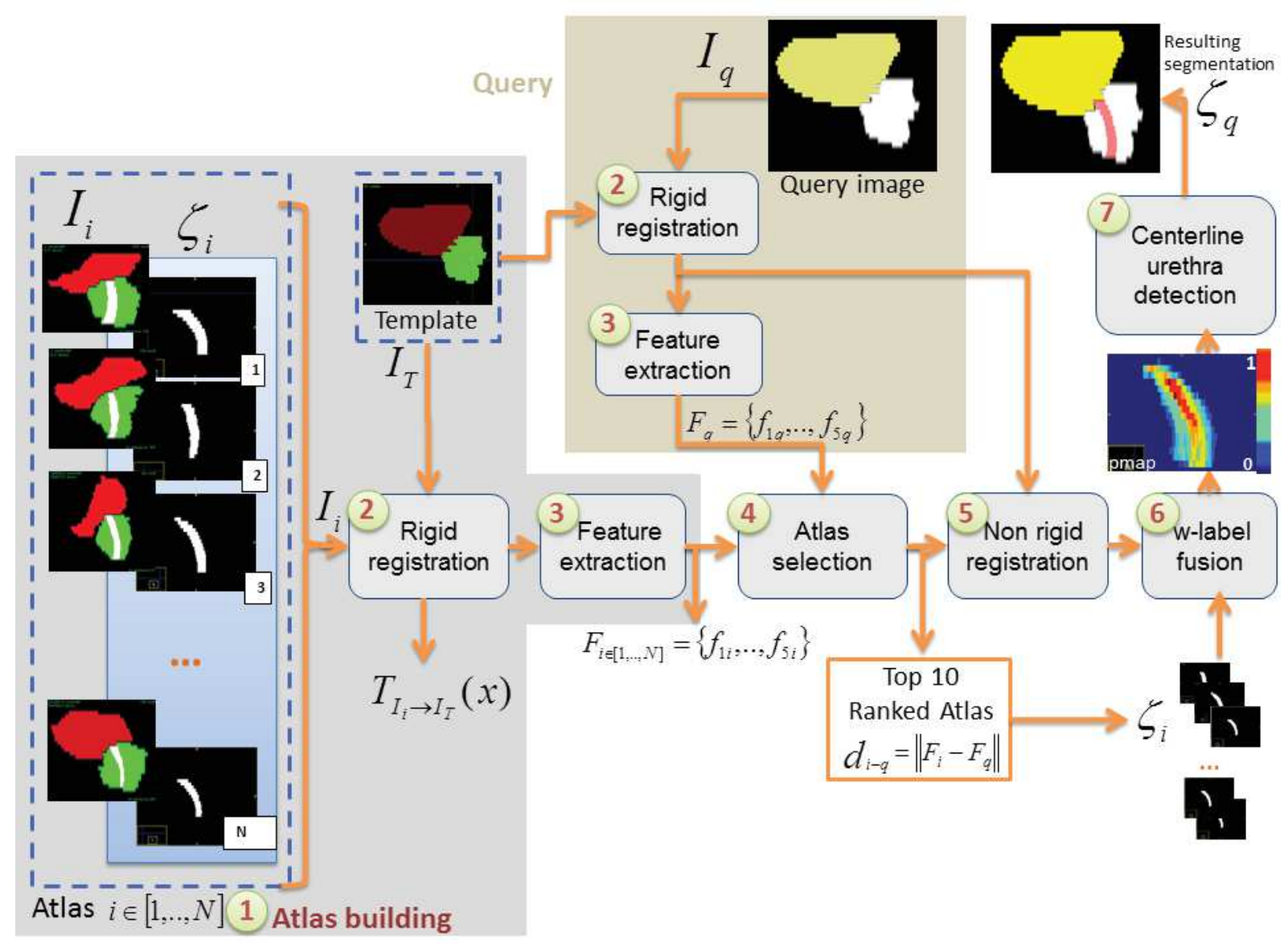

Figure 1. Overall proposed framework of multi-atlas based urethra segmentation (MABUS) method

All the individuals from the previously built atlas database (N Atlas) (steps 1) are rigidly registered to a common space $\left(I_{T}\right)$ and features are extracted (steps 2 and 3). The query image $I_{q}$ to be segmented is also rigidly aligned with the same template as the atlas database, in order to obtain a transformation $T_{I_{i} \rightarrow I_{q}}$ (steps 2 and 3). Atlas selection (step 4) followed by nonrigid registration (step 5) allow to propagate the labels (step 6) to the query image to be combined in a weighted fusion-decision step. This results in a probability map (pmap). A final centerline detection and $5 \mathrm{~mm}$ dilation yielded the segmented urethra (step 7). 


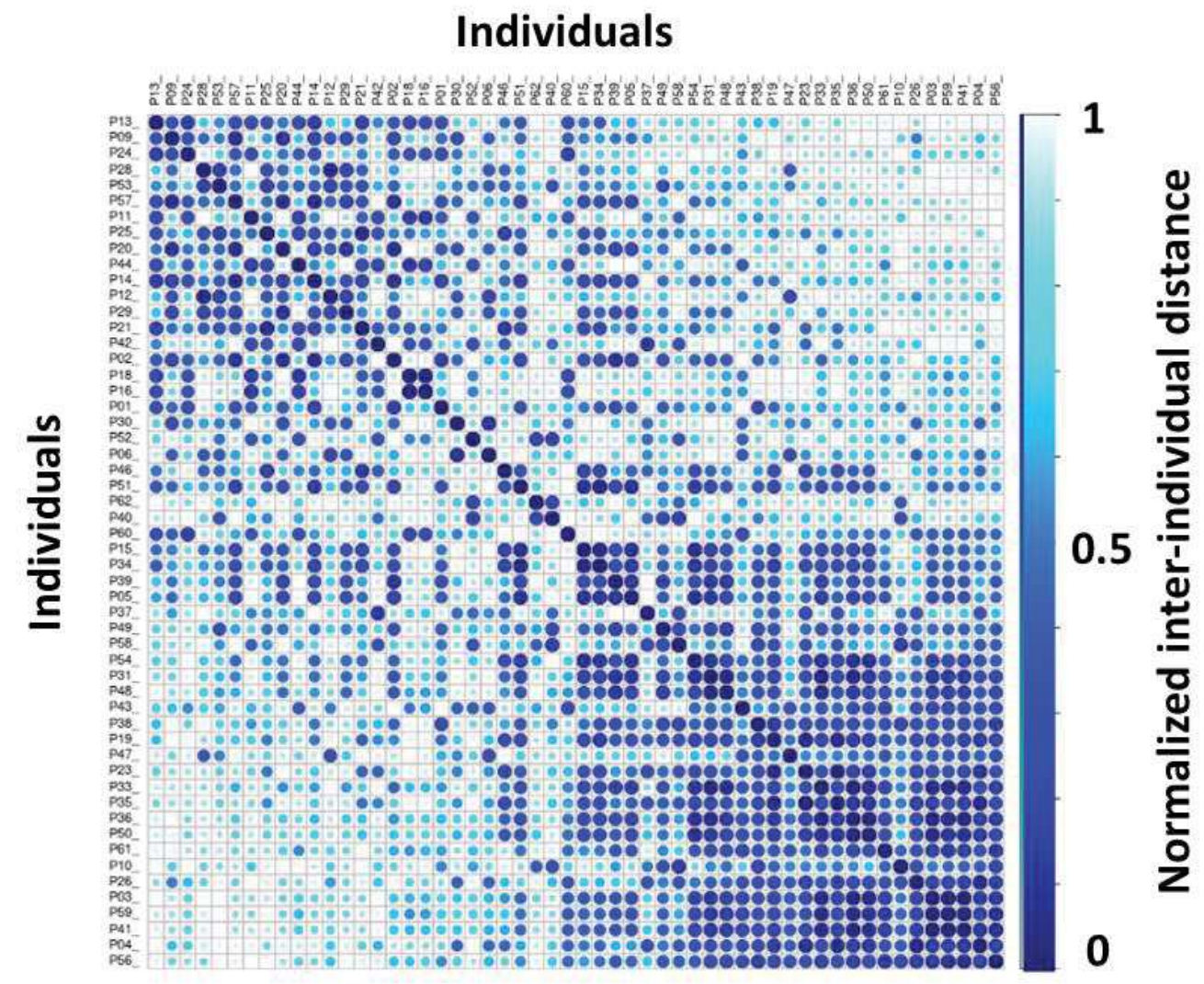

Figure 2. Inter-individual distance map

Each row represents the distance between an individual and all the others from the atlas database considering the z-score features. For each considered case there is at least $20 \%$ of individuals whose similarity is above 0.5 which may be good candidates in the atlas selection procedure. There are nevertheless some outliers for which the worst segmentations were obtained. 


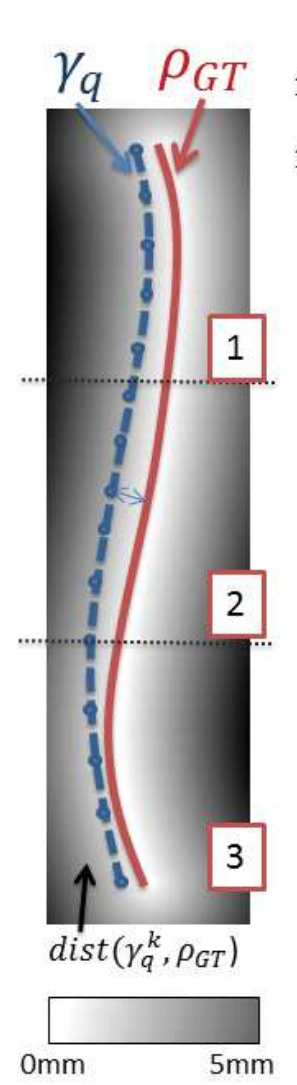

a)
Centerline Distance (CLD) [mm]
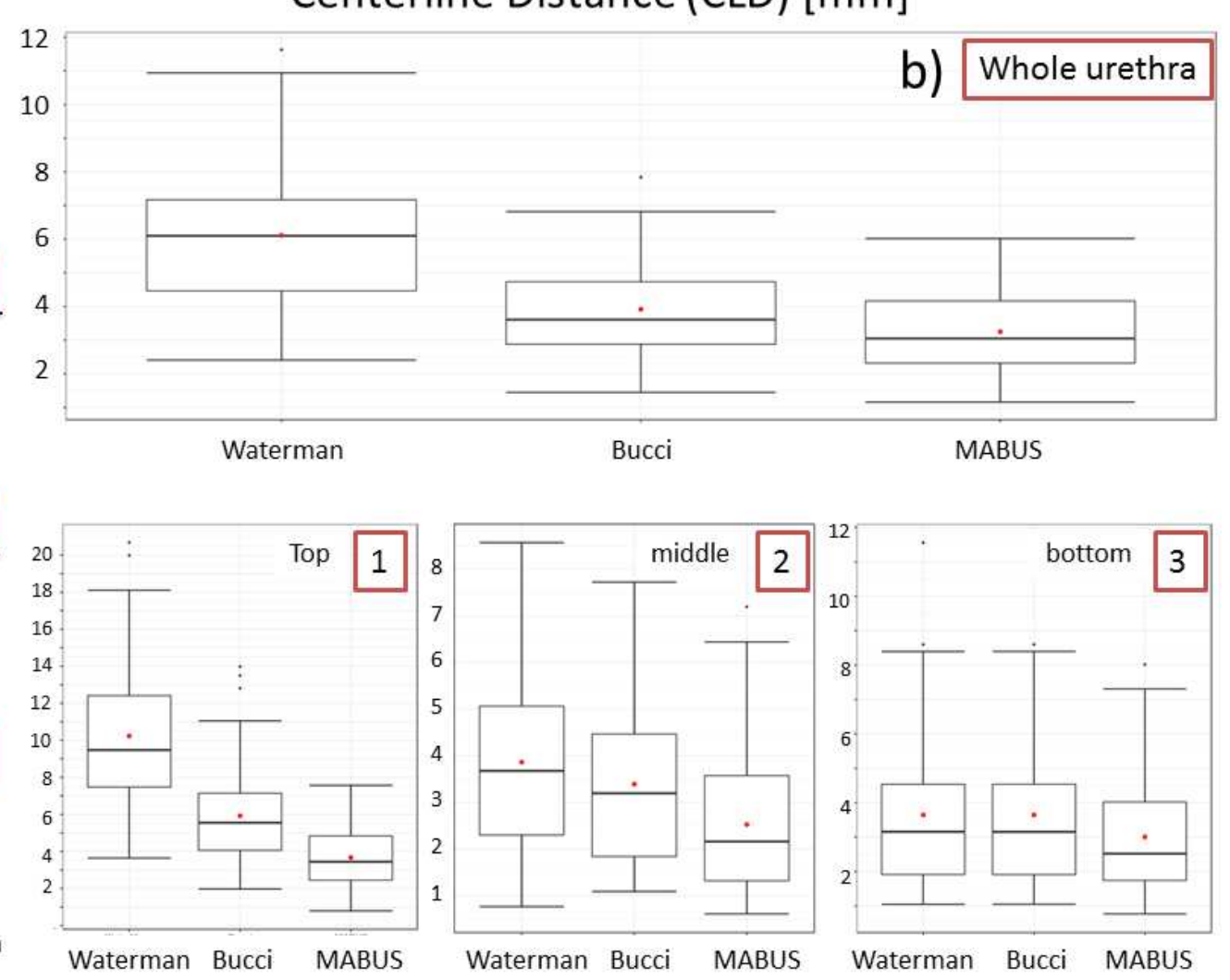

c)

Figure 3. Centerline distance (CLD) definition and leave one out validation results of the proposed method compared with previously proposed surrogate models.

a) The CLD was computed using the Euclidean distance map from the ground truth catheter centerline $\boldsymbol{\rho}_{G T}$. It was defined as the average of the distances between the ground truth catheter centerline $\rho_{G T}$ and the centerline from the segmentation $\gamma_{q} \operatorname{dist}\left(\gamma_{q}^{k}, \rho_{G T}\right)$. b) Leave one out validation results and comparison with other methods for the whole urethra and c) by urethral thirds. Box plots show the average (dot), the median (horizontal line), and the first and third quartiles. 


\section{$\%$ of centerline points (PWR)}

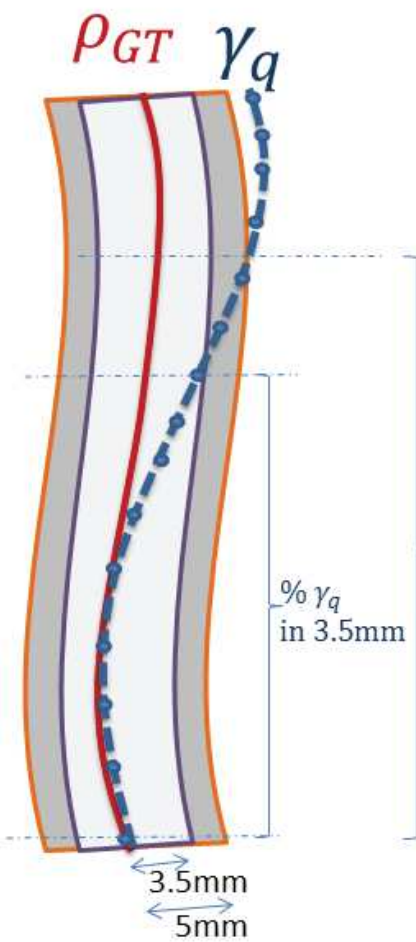

a)

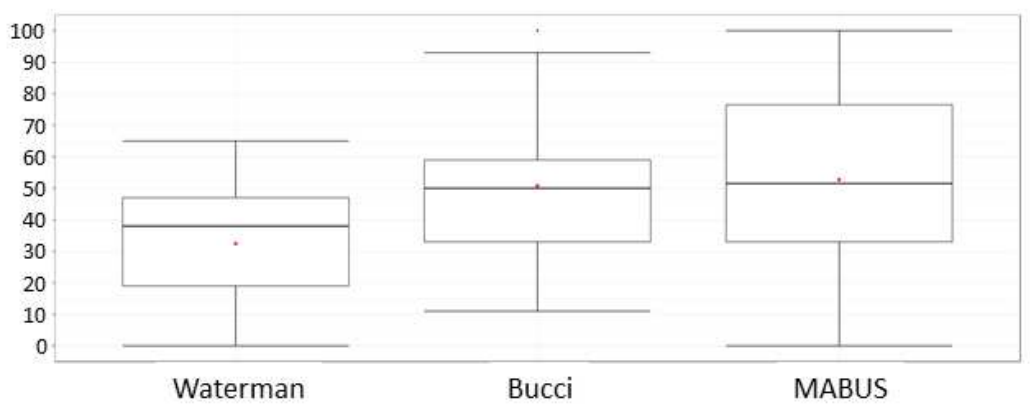

b) PWR in a radius $<3.5 \mathrm{~mm}$

in $5 \mathrm{~mm} 100$

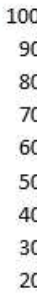

90
80
70
60
50
40
30
20
10
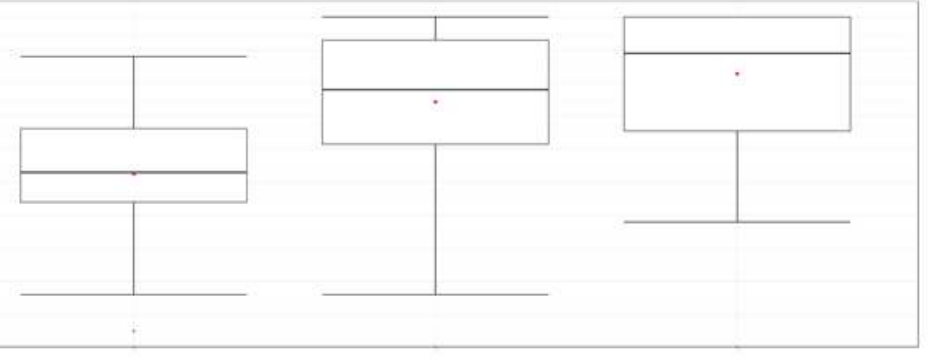

Waterman

Bucci

MABUS

C) PWR in a radius $<5 \mathrm{~mm}$

Figure 4. Percentage of segmented centerline $\left(\gamma_{q}\right)$ lying within a radius (PWR) of 3.5 or $5 \mathrm{~mm}$ around the ground truth centerline $\left(\gamma_{q}\right)$.

a) The percentage of segmented centerline lying within a radius from the ground truth centerline (PWR) was computed from the Euclidean distance map dist $\left(\gamma_{\boldsymbol{q}}^{\boldsymbol{k}}, \boldsymbol{\rho}_{\boldsymbol{G T}}\right)$. It represents the portion of the segmented $\gamma_{\boldsymbol{q}}$ within two region sizes $(<3.5 \mathrm{~mm}$ and $<5 \mathrm{~mm})$. The higher the better. b) and c) Leave one out results and comparison with the other methods. 


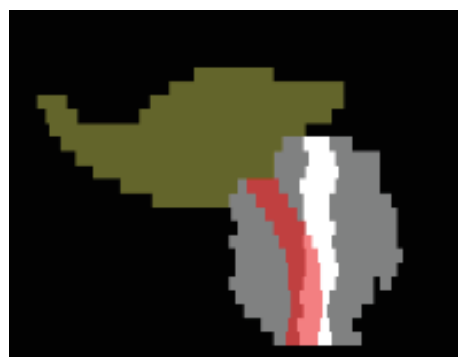

a) Waterman

$\mathrm{CLD}=6.81 \mathrm{~mm}$

PWR $<3.5 \mathrm{~mm}=19 \%$

PWR $<5 \mathrm{~mm}=50 \%$

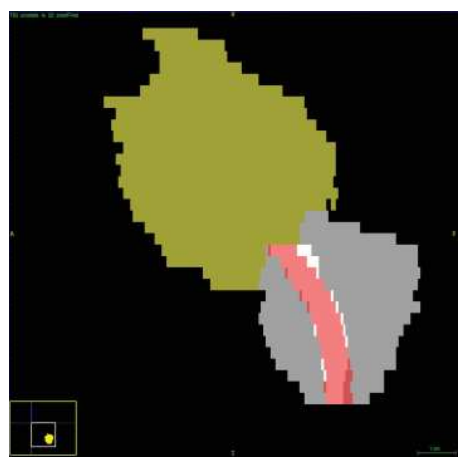

d) MABUS

$\mathrm{CLD}=1.16 \mathrm{~mm}$

PWR $<3.5 \mathrm{~mm}=100 \%$

$\mathrm{PWR}<5 \mathrm{~mm}=100 \%$

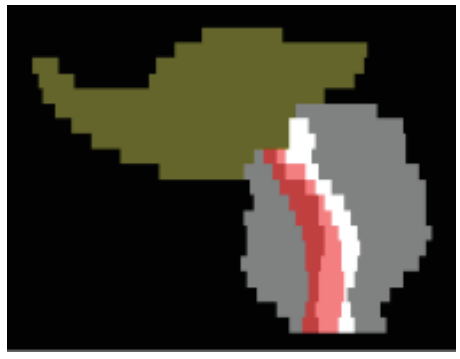

b) Bucci

$\mathrm{CLD}=4.28 \mathrm{~mm}$

$\mathrm{PWR}<3.5 \mathrm{~mm}=31 \%$

PWR $<5 \mathrm{~mm}=81 \%$

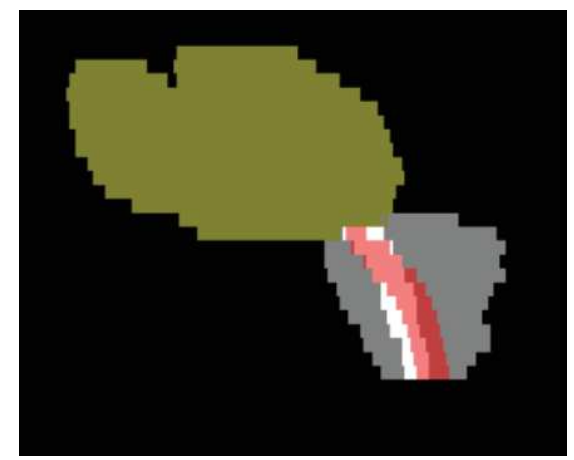

e) MABUS

$\mathrm{CLD}=3.40 \mathrm{~mm}$

$\mathrm{PWR}<3.5 \mathrm{~mm}=27 \%$

PWR $<5 \mathrm{~mm}=100 \%$

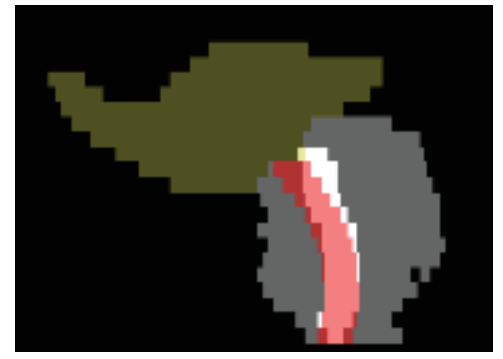

c) MABUS

CLD $=2.17 \mathrm{~mm}$

PWR $<3.5 \mathrm{~mm}=75 \%$

PWR $<5 \mathrm{~mm}=92 \%$

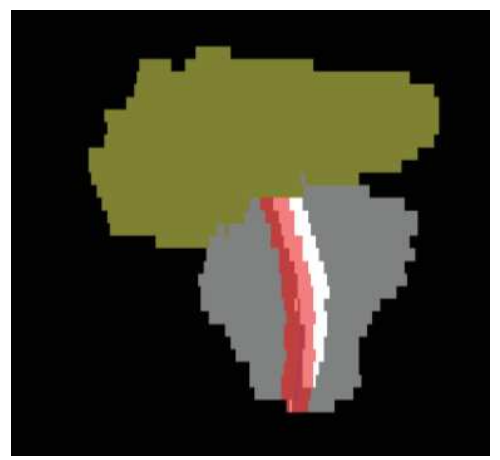

f) MABUS

$\mathrm{CLD}=2.8 \mathrm{~mm}$

PWR $<3.5 \mathrm{~mm}=50 \%$

PWR $<5 \mathrm{~mm}=100 \%$

Figure 5. Examples of urethra segmentations (white) overlaid on the actual urinary catheter (red). Top row: Comparison of MABUS with two surrogate models. Low row: different individual configurations with the proposed methodology (MABUS) only.

Urethra segmentations (white) are overlaid on the actual urinary catheter (red). Bladder is in yellow and prostate in grey. CLD: Centerline Distance; PWR: The percentage of segmented centerline lying within a radius from the ground truth centerline.

Top row shows the comparison of two surrogate models: a) Waterman and b) Bucci) with c) the proposed method (MABUS) on the same individual. Low row shows different segmentation results with MABUS. Case d) Depicts one of the best results where both centerlines almost coincide ( $C L D=1.16 \mathrm{~mm}$ ). For the two other cases e) and f) it may be noticed the nature of the accuracy errors and complimentary information brought by the considered scores (CLD and

PWR). 


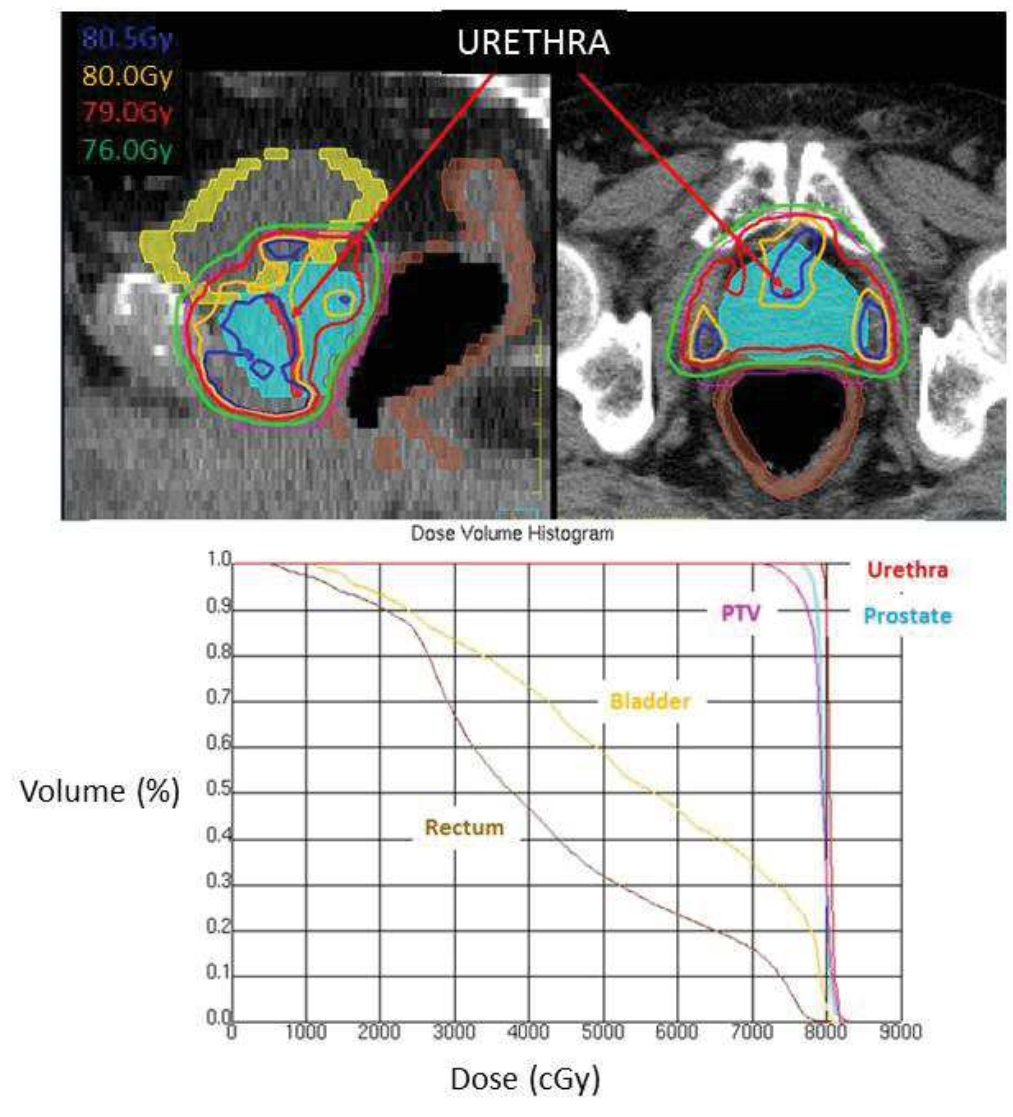

Figure 6. Example of urethra segmentation and dose distribution in IMRT planning delivering 80 Gy to the prostate

Top: CT sagittal and axial views overlaid with urethra and dose distribution. Bottom: DVH showing that the dose received by the urethra is higher than the dose received by the whole prostate (Figure A3 zooms in the region of interest). The urethra appears in red, the prostate in blue, the rectum in brown and the bladder in yellow. 